\title{
A RECURSION FORMULA FOR THE MOMENTS OF THE FIRST PASSAGE TIME OF THE ORNSTEIN-UHLENBECK PROCESS
}

\author{
DIRK VEESTRAETEN, ${ }^{*}$ University of Amsterdam
}

\begin{abstract}
In this paper we use the Siegert formula to derive alternative expressions for the moments of the first passage time of the Ornstein-Uhlenbeck process through a constant threshold. The expression for the $n$th moment is recursively linked to the lower-order moments and consists of only $n$ terms. These compact expressions can substantially facilitate (numerical) applications also for higher-order moments.
\end{abstract}

Keywords: Ornstein-Uhlenbeck process; first passage time; moments; constant threshold 2010 Mathematics Subject Classification: Primary 60J60

Secondary $60 \mathrm{~K} 30$

\section{Introduction}

The analysis of the Ornstein-Uhlenbeck process has a long tradition in various fields including astrophysics [3], neurophysiology [4], queueing theory [5], and financial mathematics [18]. The moments of the first passage time through a constant threshold are typically calculated by evaluating derivatives for the Laplace transform of the probability density function (PDF) of the first passage time (see [2], [12], [14], and [16]). Closed-form expressions have been reported for the first three moments (see [2] and [16]). The dependence on derivatives in this approach, however, implies that expressions for the moments grow substantially longer each time a higher order is to be evaluated.

Less frequently used, the Siegert formula [17] offers a recursive representation for the first passage time moments (see [1] and [11]). This is predominantly due to the cumbersome nature of the resulting integral expressions. However, calculations can be simplified considerably by rewriting integrands as series for which integrals are easier to evaluate. This simplification ultimately also allows us to obtain a recursive expression for the moments of order 3 and higher. In fact, the expression for the $n$th moment consists only of $n$ terms that each comprise one of the lower-order moments. The compact nature of these expressions should speed up computation and facilitate applications such as estimation of the parameters of a diffusion process (e.g. within the method of moments as proposed in [6] for an Ornstein-Uhlenbeck process and more recently discussed in [8]).

In Section 2 we first derive the expressions for the first and second moments after which a recursive formulation is obtained for the higher-order moments. A short application shows that the values for the skewness, kurtosis, hyperskewness, and hyperflatness quickly converge to the asymptotic values that were obtained in [13] for large thresholds. In Section 3 we use the Siegert formula [17] to additionally derive an expression for the first moment that is valid for

Received 26 July 2013; revision received 20 March 2014.

* Postal address: Amsterdam School of Economics, University of Amsterdam, Roetersstraat 11, 1018WB Amsterdam, The Netherlands. Email address: d.j.m.veestraeten@uva.nl 
the parameter range $\mu \theta \gg S$ for which the expression in Section 2 and the Laplace transform both diverge (see [10] and [15]).

\section{The recursion formula}

Let $\{X(t), t \geq 0\}$ with $X(0)=x_{0}$ be an Ornstein-Uhlenbeck process with drift $(-x / \theta+\mu)$, $\theta>0$, and infinitesimal variance $\sigma^{2}$. The process is defined on the interval $(-\infty,+\infty)$ and the threshold $S$ is an arbitrary constant with $x_{0}<S$. The Siegert formula [17] states that the moments of the first passage time through $S$ for this setup can be obtained via the following recursion expression:

$$
t_{n}\left(S \mid x_{0}\right)=n \int_{x_{0}}^{S} \frac{2 \mathrm{~d} z}{\sigma^{2} W(z)} \int_{-\infty}^{z} W(x) t_{n-1}(S \mid x) \mathrm{d} x \quad \text { for } n=1,2, \ldots,
$$

where $t_{n}\left(S \mid x_{0}\right)$ denotes the $n$th moment of the first passage time and $W(x)$ gives the steadystate PDF with

$$
W(x)=\frac{1}{\sqrt{\pi \sigma^{2} \theta}} \exp \left[-\frac{(x-\mu \theta)^{2}}{\sigma^{2} \theta}\right] .
$$

Given $t_{0}\left(S \mid x_{0}\right)=1$, the first moment, $t_{1}\left(S \mid x_{0}\right)$, can be written as

$$
t_{1}\left(S \mid x_{0}\right)=\frac{\sqrt{\pi \theta}}{\sigma} \int_{x_{0}-\mu \theta}^{S-\mu \theta} \exp \left[\frac{y^{2}}{\sigma^{2} \theta}\right]\left\{1+\operatorname{erf}\left[\frac{y}{\sqrt{\sigma^{2} \theta}}\right]\right\} \mathrm{d} y,
$$

where erf $[y]$ is the error function [7]. The evaluation of the integral in (2) will be simplified by expressing the integrand as a series in $y$. From [7, Equation 1.211.1] it follows that

$$
\exp \left[y^{2}\right]=\sum_{j=0}^{+\infty} \frac{y^{2 j}}{j !}
$$

and from [7, Equation 8.253.1] the error function is specified as

$$
\operatorname{erf}[y]=\frac{2 y}{\sqrt{\pi}} \sum_{j=0}^{+\infty} \frac{\left(-y^{2}\right)^{j}}{j !(2 j+1)} .
$$

The integrand of (2) can then be simplified accordingly into

$$
\exp \left[\frac{y^{2}}{\sigma^{2} \theta}\right]\left\{1+\operatorname{erf}\left[\frac{y}{\sqrt{\sigma^{2} \theta}}\right]\right\}=\sum_{j=0}^{+\infty} q_{j} y^{j}
$$

with

$$
q_{j}= \begin{cases}\left(\frac{2}{\sqrt{\pi \sigma^{2} \theta}}\right)^{j} & \text { for } j=0 \text { and } j=1, \\ \frac{2}{j \sigma^{2} \theta} q_{j-2} & \text { for } j \geq 2 .\end{cases}
$$

The first moment, $t_{1}\left(S \mid x_{0}\right)$, is given by

$$
t_{1}\left(S \mid x_{0}\right)=\frac{\sqrt{\pi \theta}}{\sigma} \sum_{j=0}^{+\infty} \frac{q_{j}}{j+1}\left\{(S-\mu \theta)^{j+1}-\left(x_{0}-\mu \theta\right)^{j+1}\right\} .
$$


From (1) and straightforward but lengthy calculations the second moment, $t_{2}\left(S \mid x_{0}\right)$, is specified as

$$
\begin{aligned}
t_{2}\left(S \mid x_{0}\right)= & \frac{2}{\sigma} t_{1}\left(S \mid x_{0}\right) \sum_{j=0}^{+\infty} \frac{q_{j}}{j+1}\left\{\sqrt{\pi \theta}(S-\mu \theta)^{j+1}-\frac{2}{\sigma} s_{1, j}\right\} \\
& -\frac{4 \sqrt{\pi \theta}}{\sigma^{3}} \sum_{j=0}^{+\infty} \frac{q_{j}}{j+1}\left\{r_{2, S-\mu \theta, j}-r_{2, x_{0}-\mu \theta, j}\right\}
\end{aligned}
$$

where

$$
\begin{aligned}
& r_{2, y, j}= \begin{cases}-\frac{\sigma^{2} \theta y^{j+1}}{2(j+1)} & \text { for } j=0 \text { and } j=1, \\
-\frac{\sigma^{2} \theta y^{j+1}}{2(j+1)}+\frac{j \sigma^{2} \theta}{2} r_{2, y, j-2} & \text { for } j \geq 2,\end{cases} \\
& s_{1, j}= \begin{cases}\frac{j \sqrt{\pi}\left(\sigma^{2} \theta\right)^{3 / 2}}{4} & \text { for } j=0 \text { and } j=1, \\
\frac{j \sigma^{2} \theta}{2} s_{1, j-2} & \text { for } j \geq 2 .\end{cases}
\end{aligned}
$$

The expressions for the first two moments in (3) and (4) are more compact when compared with the relations derived in [2], [12], [14], and [16]. Moreover, the recursive nature of these expressions and especially of the coefficients $q, r$, and $s$ has the advantage of allowing us to also specify the higher-order moments in rather compact and recursive form. In fact, the third moment, $t_{3}\left(S \mid x_{0}\right)$, is computed as

$$
\begin{aligned}
t_{3}\left(S \mid x_{0}\right)= & \frac{3}{\sigma} t_{2}\left(S \mid x_{0}\right) \sum_{j=0}^{+\infty} \frac{q_{j}}{j+1}\left\{\sqrt{\pi \theta}(S-\mu \theta)^{j+1}-\frac{2}{\sigma} s_{1, j}\right\} \\
& -\frac{12}{\sigma^{3}} t_{1}\left(S \mid x_{0}\right) \sum_{j=0}^{+\infty} \frac{q_{j}}{j+1}\left\{\sqrt{\pi \theta} r_{2, S-\mu \theta, j}-\frac{2}{\sigma} s_{2, j}\right\} \\
& +\frac{24 \sqrt{\pi \theta}}{\sigma^{5}} \sum_{j=0}^{+\infty} \frac{q_{j}}{j+1}\left\{r_{3, S-\mu \theta, j}-r_{3, x_{0}-\mu \theta, j}\right\}
\end{aligned}
$$

with

$$
\begin{aligned}
& r_{3, y, j}= \begin{cases}-\frac{\sigma^{2} \theta}{2(j+1)} r_{2, y, j} & \text { for } j=0 \text { and } j=1, \\
-\frac{\sigma^{2} \theta}{2(j+1)} r_{2, y, j}+\frac{j \sigma^{2} \theta}{2} r_{3, y, j-2} & \text { for } j \geq 2,\end{cases} \\
& s_{2, j}= \begin{cases}-\frac{j \sigma^{2} \theta}{2(j+1)} s_{1, j} & \text { for } j=0 \text { and } j=1, \\
-\frac{\sigma^{2} \theta}{2(j+1)} s_{1, j}+\frac{j \sigma^{2} \theta}{2} s_{2, j-2} & \text { for } j \geq 2 .\end{cases}
\end{aligned}
$$


Employing (1) for subsequent orders reveals that the moments of order 3 and higher can be specified as

$$
\begin{aligned}
t_{n}\left(S \mid x_{0}\right)= & \frac{n}{\sigma} t_{n-1}\left(S \mid x_{0}\right) \sum_{j=0}^{+\infty} \frac{q_{j}}{j+1}\left\{\sqrt{\pi \theta}(S-\mu \theta)^{j+1}-\frac{2}{\sigma} s_{1, j}\right\} \\
& +\sum_{k=3}^{n} v_{n, k} t_{n-k+1}\left(S \mid x_{0}\right) \sum_{j=0}^{+\infty} \frac{q_{j}}{j+1}\left\{\sqrt{\pi \theta} r_{k-1, S-\mu \theta, j}-\frac{2}{\sigma} s_{k-1, j}\right\} \\
& +w_{n} \sum_{j=0}^{+\infty} \frac{q_{j}}{j+1}\left\{r_{n, S-\mu \theta, j}-r_{n, x_{0}-\mu \theta, j}\right\} \quad \text { for } n=3,4, \ldots,
\end{aligned}
$$

where

$$
\begin{aligned}
& r_{n, y, j}= \begin{cases}-\frac{\sigma^{2} \theta}{2(j+1)} r_{n-1, y, j} & \text { for } j=0, j=1, \text { and } n \geq 3, \\
-\frac{\sigma^{2} \theta}{2(j+1)} r_{n-1, y, j}+\frac{j \sigma^{2} \theta}{2} r_{n, y, j-2} & \text { for } j \geq 2 \text { and } n \geq 3,\end{cases} \\
& s_{n, j}= \begin{cases}-\frac{j \sigma^{2} \theta}{2(j+1)} s_{n-1, j} & \text { for } j=0, j=1, \text { and } n \geq 3, \\
-\frac{\sigma^{2} \theta}{2(j+1)} s_{n-1, j}+\frac{j \sigma^{2} \theta}{2} s_{n, j-2} & \text { for } j \geq 2 \text { and } n \geq 3,\end{cases} \\
& v_{n, k}= \begin{cases}-\frac{2 n(n-1)}{\sigma^{3}} & \text { for } k=3 \text { and } n \geq 3, \\
-\frac{2 n}{\sigma^{2}} v_{n-1, k-1} \quad \text { for } k \geq 4 \text { and } n \geq 3,\end{cases} \\
& w_{n}= \begin{cases}\frac{24 \sqrt{\pi \theta}}{\sigma^{5}} & \text { for } n=3, \\
-\frac{2 n}{\sigma^{2}} w_{n-1} & \text { for } n \geq 4 .\end{cases}
\end{aligned}
$$

The specifications for the first and second moments in (3) and (4), respectively, and the recursion formula in (5) for order 3 and higher thus offer relatively neat and compact expressions that should reduce computational burden and facilitate applications. It should be noted that the recursion formula as well as the expressions for the (higher-order) moments obtained via the Laplace transform are valid for the same range of parameters as they both rely on sums in which essentially the same terms are raised to positive and increasing powers.

The above results can be used to illustrate the effects on (standardized) moments of increasing $S$ (see [11] and [13]). The first six moments are connected to the mean, variance, skewness, kurtosis, hyperskewness, and hyperflatness via the following relations:

$$
\begin{aligned}
& \text { mean }=t_{1}\left(S \mid x_{0}\right), \\
& \text { variance }=\mu_{2}=t_{2}\left(S \mid x_{0}\right)-t_{1}^{2}\left(S \mid x_{0}\right), \\
& \text { skewness }=\frac{1}{\mu_{2}^{3 / 2}}\left\{t_{3}\left(S \mid x_{0}\right)-3 t_{1}\left(S \mid x_{0}\right) t_{2}\left(S \mid x_{0}\right)+2 t_{1}^{3}\left(S \mid x_{0}\right)\right\},
\end{aligned}
$$




$$
\begin{aligned}
& \text { kurtosis }=\frac{1}{\mu_{2}^{2}}\left\{t_{4}\left(S \mid x_{0}\right)-4 t_{1}\left(S \mid x_{0}\right) t_{3}\left(S \mid x_{0}\right)+6 t_{1}^{2}\left(S \mid x_{0}\right) t_{2}\left(S \mid x_{0}\right)-3 t_{1}^{4}\left(S \mid x_{0}\right)\right\}, \\
& \text { hyperskewness }=\frac{1}{\mu_{2}^{5 / 2}}\left\{t_{5}\left(S \mid x_{0}\right)-5 t_{1}\left(S \mid x_{0}\right) t_{4}\left(S \mid x_{0}\right)+10 t_{1}^{2}\left(S \mid x_{0}\right) t_{3}\left(S \mid x_{0}\right)\right. \\
& \left.\quad \quad-10 t_{1}^{3}\left(S \mid x_{0}\right) t_{2}\left(S \mid x_{0}\right)+4 t_{1}^{5}\left(S \mid x_{0}\right)\right\}, \\
& \text { hyperflatness }=\frac{1}{\mu_{2}^{3}}\left\{t_{6}\left(S \mid x_{0}\right)-6 t_{1}\left(S \mid x_{0}\right) t_{5}\left(S \mid x_{0}\right)+15 t_{1}^{2}\left(S \mid x_{0}\right) t_{4}\left(S \mid x_{0}\right)\right. \\
& \left.\quad-20 t_{1}^{3}\left(S \mid x_{0}\right) t_{3}\left(S \mid x_{0}\right)+15 t_{1}^{4}\left(S \mid x_{0}\right) t_{2}\left(S \mid x_{0}\right)-5 t_{1}^{6}\left(S \mid x_{0}\right)\right\} .
\end{aligned}
$$

It was noted in [11] that the numerical values for the skewness in [2] approached 2 for large values of $S$. In fact, [11] showed that an exponential PDF approximates the first passage-time PDF well for large thresholds. Subsequently, [13] proved that $t_{n}\left(S \mid x_{0}\right) \sim n ![g(S)]^{n}$ for $n=1,2, \ldots$ for large $S$ with $g(S)=(S / \sqrt{2 \pi}) \exp \left[-S^{2} / 2\right]$ such that the skewness indeed moves to 2 when $S$ increases. Likewise, the kurtosis, hyperskewness, and hyperflatness approach 9, 44, and 265, respectively, when $S$ becomes large. Convergence to these values is confirmed from Figure 1, in which the latter standardized moments are scaled by their asymptotic values for the parameter values $\theta=20, \mu=0.5, \sigma^{2}=10$, and $x_{0}=3$. As required, the ratios (quickly) decrease to 1 but can be sizeable for smaller boundaries.

\section{A series expression for the first moment for $\mu \theta \gg S$}

As discussed in, for example, [10] and [15], the Siegert formula and the Laplace transform fail to produce reliable results for the first moment in the parameter range $\mu \theta \gg S$. Indeed, the first moment in (3) diverges on account of the presence of large terms that are raised to positive and increasing powers. This divergence finds its origin in the fact that the series expansion for the error function in Section 2 produces inaccurate results for highly negative arguments. However, [7, Equation 8.254] and the properties of the error function offer the

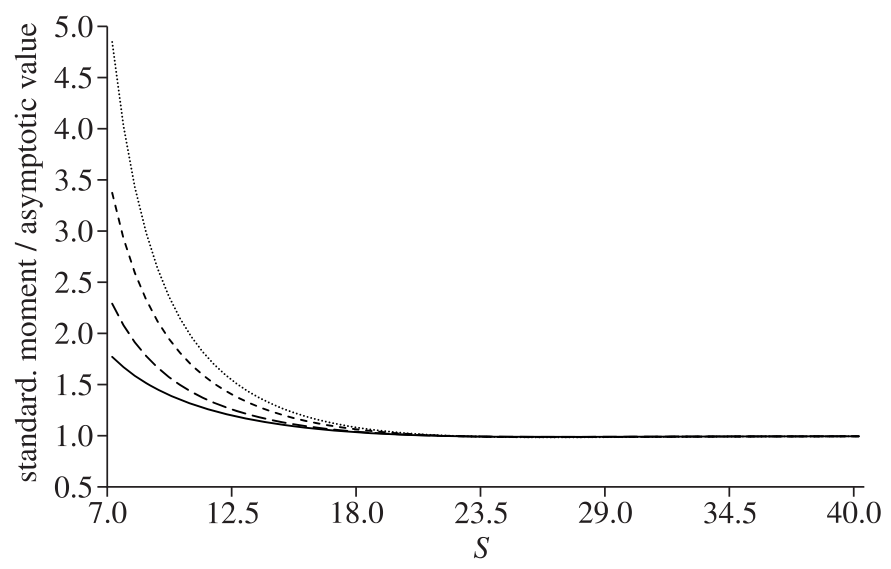

FiguRe 1: Standardized moments scaled by their asymptotic value for large values of the threshold $S$ : skewness (solid line), kurtosis (broad dashed line), hyperskewness (dashed line), and hyperflatness (dotted line) for the parameter values $\theta=20, \mu=0.5, \sigma^{2}=10$, and $x_{0}=3$. 
following alternative expansion for large negative values of the argument

$$
\operatorname{erf}[y]=-1-\frac{\exp \left[-y^{2}\right]}{y \sqrt{\pi}} \sum_{j=0}^{p} \frac{(-1)^{j}(2 j-1) ! !}{\left(2 y^{2}\right)^{j}}
$$

where $(2 j-1) ! !=(2 j-1)(2 j-3) \cdots 3 \cdot 1$ and $(-1) ! !=1 ! !=1$. For notational brevity, the term $O|y|^{-2 p-y}$ is omitted as the expression below for the first moment is extremely accurate even for small values of $p$. The integrand in (2) then can be rewritten as

$$
\exp \left[\frac{y^{2}}{\sigma^{2} \theta}\right]\left\{1+\operatorname{erf}\left[\frac{y}{\sqrt{\sigma^{2} \theta}}\right]\right\}=\frac{1}{\sqrt{\pi}} \sum_{j=0}^{p} \frac{(-1)^{j+1}(2 j-1) ! !\left(\sigma^{2} \theta\right)^{j+1 / 2}}{2^{j} y^{2 j+1}},
$$

which allows the first moment to be specified as

$$
t_{1}\left(S \mid x_{0}\right)=-\theta \ln \left[\frac{S-\mu \theta}{x_{0}-\mu \theta}\right]+\theta \sum_{j=1}^{p} \frac{(-1)^{j}(2 j-1) ! !}{j 2^{j+1}}\left\{\left(\frac{S-\mu \theta}{\sigma \sqrt{\theta}}\right)^{-2 j}-\left(\frac{x_{0}-\mu \theta}{\sigma \sqrt{\theta}}\right)^{-2 j}\right\} .
$$

The expression in (6) comprises two terms of which the first has been discussed in [10] as the first moment for $\sigma \rightarrow 0$, i.e. for the deterministic case. The sum in (6) then represents the added effect of nonzero $\sigma$ on $t_{1}\left(S \mid x_{0}\right)$ and as required reduces to 0 for $\sigma \rightarrow 0$. The first moment in (6) then offers an alternative to the ad hoc method for nonzero $\sigma$ that was proposed in [9].

Numerical examinations showed that the expression for $t_{1}\left(S \mid x_{0}\right)$ in (6) clearly is to be preferred over (3) for values of $((S-\mu \theta) / \sigma \sqrt{\theta})$ and $\left(\left(x_{0}-\mu \theta\right) / \sigma \sqrt{\theta}\right)$ below -5 . Indifference between the two expressions arises for values between -4 and -5 , whereas the expression in (3) is superior for values that exceed -4 .

\section{Acknowledgement}

I would like to sincerely thank the anonymous referee for the constructive suggestions that substantially improved this paper.

\section{References}

[1] Capocelli, R. M. ANd Ricciardi, L. M. (1971). Diffusion approximation and first passage time problem for a model neuron. Kybernetik (Berlin) 8, 214-223.

[2] Cerbone, G., Ricciardi, L. M. and Sacerdote, L. (1981). Mean, variance and skewness of the first passage time for the Ornstein-Uhlenbeck process. Cybernet. Systems 12, 395-429.

[3] Chandrasekhar, S. (1943). Dynamical friction. II. The rate of escape of stars from clusters and the evidence for the operation of dynamical friction. Astrophys. J. 97, 263-273.

[4] Gluss, B. (1967). A model for neuron firing with exponential decay of potential resulting in diffusion equations for probability density. Bull. Math. Biophys. 29, 233-243.

[5] IgLEHART, D. L. (1965). Limiting diffusion approximations for the many server queue and the repairman problem. J. Appl. Prob. 2, 429-441.

[6] Inoue, J., Sato, S. And Ricciardi, L. M. (1995). On the parameter estimation for diffusion models of single neuron's activities. Biol. Cybern. 73, 209-221.

[7] Jeffrey, A. ANd Zwillinger, D. (eds) (2000). Table of Integrals, Series, and Products, 6th edn. Academic Press, San Diego, CA.

[8] LÁnský, P. And Ditlevsen, S. (2008). A review of the methods for signal estimation in stochastic diffusion leaky integrate-and-fire neuronal models. Biol. Cybern. 99, 253-262.

[9] LÁNSKÝ, P. AND SACERDOTE, L. (2001). The Ornstein-Uhlenbeck neuronal model with signal-dependent noise. Phys. Lett. A 285, 132-140. 
[10] LÁnský, P., Sacerdote, L. And Tomassetti, F. (1995). On the comparison of Feller and Ornstein-Uhlenbeck models for neural activity. Biol. Cybern. 73, 457-465.

[11] Nobile, A. G., Ricciardi, L. M. and Sacerdote, L. (1985). Exponential trends of Ornstein-Uhlenbeck firstpassage-time densities. J. Appl. Prob. 22, 360-369.

[12] Ricciardi, L. M. And SACERDote, L. (1979). The Ornstein-Uhlenbeck process as a model for neuronal activity. Biol. Cybern. 35, 1-9.

[13] Ricciardi, L. M. and SATo, S. (1988). First-passage-time density and moments of the Ornstein-Uhlenbeck process. J. Appl. Prob. 25, 43-57.

[14] Roy, B. K. AND Smith, D. R. (1969). Analysis of the exponential decay model of the neuron showing frequency threshold effects. Bull. Math. Biophys. 31, 341-357.

[15] Sacerdote, L. and Giraudo, M. T. (2013). Stochastic integrate and fire models: a review on mathematical methods and their applications. In Stochastic Biomathematical Models, Springer, Heidelberg, pp. 99-148.

[16] Sato, S. (1978). On the moments of the firing interval of the diffusion approximated model neuron. Math. Biosci. 39, 53-70.

[17] Siegert, A. J. F. (1951). On the first passage time probability problem. Phys. Rev. (2) 81, 617-623.

[18] VAsiceK, O. (1977). An equilibrium characterization of the term structure. J. Financ. Econ. 5, 177-188. 